\title{
Knowledge sharing as an imperative antecedent to organizational performance: An empirical study of IT organizations
}

\author{
Effulgence \\ Vol. 17, No. 2 (Special Issue) \\ July - December 2019 \\ Rukmini Devi Institute of Advanced Studies \\ E-mail : effulgence@rdias.ac.in, Website : www.rdias.ac.in \\ http://effulgence.rdias.ac.in/user/default.aspx \\ https://dx.doi.org/10.33601/effulgence.rdias/v17/iSpl2/2019/1-20
}

\author{
Dr. Karishma Gulati Trehan ${ }^{1}$
}

\begin{abstract}
In the dynamic phase of the market, knowledge-sharing inclination is demarcated as the capability to deliver, contact, and formulate oneself to contribute in knowledge-sharing accomplishments. This encompasses the organization's competence in associating and apportioning capitals for improvement of employees and management systems to assist in knowledge sharing. The purpose of this study is to shed more light into the relationship of Tacit and Explicit knowledge sharing and various dimensions of organizational performance based on empirical research, conducted in IT organizations. A structured instrument was developed based on the review of the literature of the theme of research for data collection from the employees of top 5 IT organizations (as per NASSCOM 2016-17). The present research provides an exclusive theoretical model that demonstrates the impact of Tacit and Explicit knowledge sharing in Customer metric organizational performance (COP), Process metric organizational performance (POP), People metric organizational performance (PLOP) and Financial metric organizational performance (FOP). It is concluded that if an organization wants to increase their performance they need to have a balance between the Tacit and Explicit knowledge.
\end{abstract}

Keywords: Tacit knowledge sharing; Explicit knowledge sharing; Customer metric organizational performance; Process metric organizational performance; People metric organizational performance; Financial metric organizational performance.

1. Assistant Professor, Vivekananda School of Business Studies, Vivekananda Institute of Professional Studies (Affiliated to GGSIPU), dr.karishmagulatitrehan@gmail.com 


\section{INTRODUCTION}

$\mathrm{I}_{\mathrm{s}}^{\mathrm{t}}$ is not dubious to say that if an organization stimulates knowledge sharing and implement that valuable knowledge amid it's employees it can attain competitive edge (Nonaka et al., 2000, Tuntrabundit and Tuntrabundit, 2011).

The two foremost categories of knowledge are Tacit knowledge and Explicit knowledge (Fey and Furu, 2008; Hedlund, 1994; Nonaka and Takeuchi, 1995; Polanyi, 1966; Nonaka, 1994; Spender, 1996; Tsoukas, 1996; Roberts, 2000;). Knowledge sharing may transpire at the discrete level; though, to attain ingenuity, an organization should manage it appropriately, captivating the factors like, organizational structure, actions, strategies, and culture. Knowledge sharing possesses diverse components: its documentation and tools for transmission, procurement and accretion, and assimilation and dissemination (Ipe, 2003). Argote and Ingram (2000) and Boh (2007) explains knowledge sharing as equally formal and informal process for sharing, assimilating, understanding, relating, and entrenching knowledge in employees and teams.

Among the employees, knowledge-sharing inclination is demarcated as the capability to deliver, contact, and formulate oneself to contribute in knowledge-sharing accomplishments. This encompasses the organization's competence in associating and apportioning capitals for improvement of employees and management systems to assist in knowledge sharing.

\section{LITERATURE REVIEW}

Knowledge sharing cannot materialize if deprived of sustenance from organizations (Al-Alawi et al., 2007; Connelly and Kelloway, 2003; Darroch, 2005; Lin, 2006; Lin, 2007; Lin and Lee, 2004; Wasko and Faraj, 2000; and Wasko and Faraj, 2005). For expediting the foundation of innovative knowledge, knowledge sharing facilitates the organization to retort rapidly and competently to the variations in the business environment (Argote and Ingram, 2000; Kamasak and Bulutlar, 2010). competitive advantage is the result of upgraded organizational performance which in turn is the outcome of efficacious knowledge sharing (Lee, 2001; Cheng, 2011). Sharing processes, though, do not assure that knowledge is utilised in escalation of organizational performance (Chen et al., 2006). The vital aspects for the foundation and preservation of any competitive advantage are distinguishing, acquiring and meritoriously employing original knowledge (Martin-De Castro et al., 2011). To create innovative products and services, organizations should focus on incorporating knowledge from peripheral bodies along with their prevailing stock of knowledge, (Todorova and Durisin, 2007; Martelo-Landroguez and Cegarra-Navarro, 2014).

The connotation of knowledge sharing in organizations is exceedingly accredited nowadays and numerous establishments have started refining the knowledge sharing inside and crosswise the departments (Berends, 2005). The knowledge sharing has been implicated as a construct and expressed contrarily in the research works (Yoo et al., 2007). Matin et al. (2010) opine that "Knowledge sharing includes processes by which knowledge flows amid resource and receiver. Each person or organizational unit can be a source, sender, facilitator or mediator amid source and receiver".

The literature recommends that top management sustenance is certainly allied with knowledge sharing (Gupta, 2008; and Hsu and Wang, 2008). This is because, knowledge sharing is associated to organizational performance, and diverse parameters of knowledge sharing subsidize the performance in different ways. Conditional elements (like incorporation of events, organizational structure and features of top management) stimuli the association amid knowledge sharing and performance (Du et al., 2007). Knowledge sharing positively affects the organizational performance, and organizations need to take advantage of knowledge sharing to 
incorporate innovation (Hoffman et al., 2005; Yang, 2005; Du et al., 2007; Cheng et al., 2008; Hsu, 2008; Ngah and Ibrahim, 2010; Wang and Wang, 2012 and Wu et al., 2012). Antecedent studies on the paradigm of organizational performance divulges that there is no agreement amongst the investigators on the suitable parameters of organization performance. As a consequence, an extensive assortment of performance parameters, i.e., objective and subjective parameters, as well as financial and nonfinancial parameters are utilised in most of the research studies (Vij and Bedi, 2012; Covin and Slevin, 1989; Ford \& Staples, 2010; Koch, 2011; Dess and Robinson, 1984; Uncles, 2011).

\section{RESEARCH GAP AND OBJECTIVES}

The conception of knowledge sharing as a construct has been developed in the preceding era and examiners have reconnoitred it in varied standpoints (Burke, 2011; Chen, Huang \& Hsiao, 2010; Sugarman, 2010). There is plentiful research work on the role knowledge sharing in organizational performance, but there is no evidence on the investigation of the which type of knowledge Tacit or explicit have more influence on organizational performance (Matzler et al., 2011; Ogunseye et al., 2011; Wang, 2004). Also it is not investigated in antecedent studies that which form of tacit and explicit will influence more on organizational performance. In most of the studies, organizational performance is taken as an individual construct and the various dimensions of organizational performance are not articulated. Therefore, the research question for the present study is:
"Do Tacit and Explicit knowledge sharing affects various dimensions of organizational performance in IT organisations in India?"

To study this research question in depth, following research objectives have been formulated:

a) To analyse the significant difference between the TKS and customer metric organizational performance of IT organizations in India.

b) To analyse the significant difference between the TKS and people metric organizational performance of IT organizations in India.

c) To analyse the significant difference between the TKS and process metric organizational performance of IT organizations in India.

d) To analyse the significant difference between the TKS and financial metric organizational performance of IT organizations in India.

e) To analyse the significant difference between the EKS and customer metric organizational performance of IT organizations in India.

f) To analyse the significant difference between the EKS and people metric organizational performance of IT organizations in India.

g) To analyse the significant difference between the EKS and process metric organizational performance of IT organizations in India.

h) To analyse the significant difference between the EKS and financial metric organizational performance of IT organizations in India.

1. Conceptual framework and development of hypotheses 


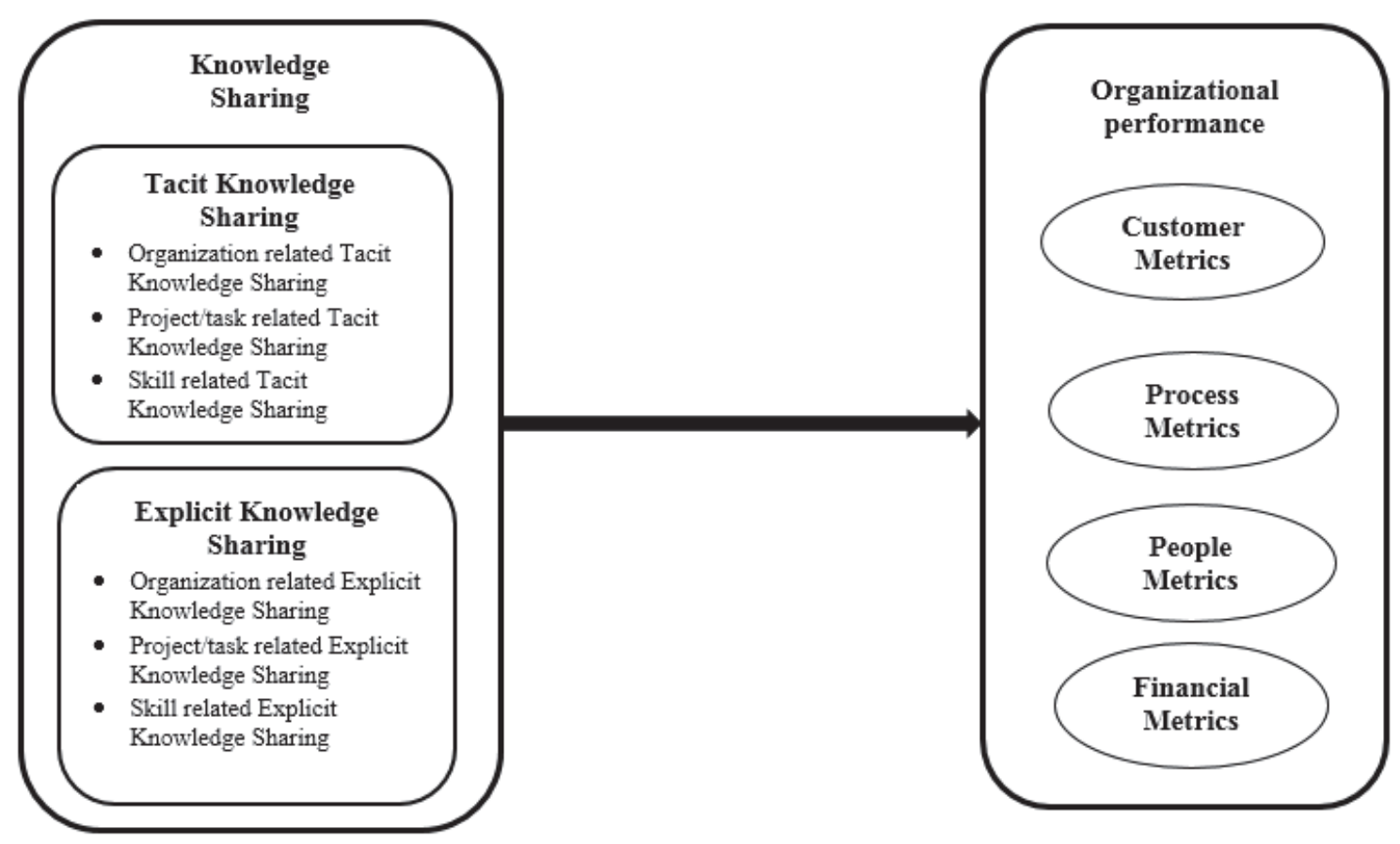

Figure 1: Conceptual Framework for role of knowledge sharing in organizational performance

The study involves two independent variables Tacit Knowledge Sharing (TKS) and Explicit KS (EKS); and organizational performance as dependent variable. Tacit and Explicit KS has three dimensions each: Organisation related Tacit KS (OTKS), Project/task related Tacit KS (PTKS), Skill related Tacit KS (STKS), Organisation related Explicit KS (OEKS), Project/task related Explicit KS (PEKS) and Skill related Explicit KS (SEKS). Organizational performance has four dimensions - Customer metric organizational performance (COP), Process metric organizational performance (POP), People metric organizational performance (PLOP) and Financial metric organizational performance (FOP).

Following hypotheses are developed:

H01: No substantial variance exists in the TKS and Customer metric organizational performance of the personnel of IT organisations in India.

H02: No substantial variance exists in the TKS and Process metric organizational performance of the personnel of IT organisations in India.

H03: No substantial variance exists in the TKS and People metric organizational performance of the personnel of IT organisations in India.

H04: No substantial variance exists in the TKS and Financial metric organizational performance of the personnel of IT organisations in India.

H05: No substantial variance exists in the EKS and Customer metric organizational performance of the personnel of IT organisations in India.

H06: No substantial variance exists in the EKS and People metric organizational performance of the personnel of IT organisations in India.

H07: No substantial variance exists in the EKS and Process metric organizational performance of the personnel of IT organisations in India.

H08: No substantial variance exists in the EKS and Financial metric organizational performance of the personnel of IT organisations in India.

\section{METHODOLOGY}

\section{Sample}

The sample was drawn from employees working at top 5 IT organisations as per NASSCOM 2016-17 using a multistage stratified sampling. Of the total IT 
organizations as per NASSCOM 2016-17, in the first stage top 5 IT organisations are selected. In the second stage, 5 primary departments of the organization i.e. Marketing, Finance, HR, IT and Operations are selected. In the next stage, the respondents for this study were selected randomly, from employees working in IT organisations, who are really associated with knowledge sharing process in the organizations. Following these procedures, out of 400, 362 usable responses are collected (response rate $=90.5 \%)$.

\section{Demographic profile of respondents}

Of the 362 respondents' majority were males (55.2\%) as compared to females (44.8\%). Most of the respondents those participated in the study are from HR department (23.1\%) and IT department (22.2\%). The respondents from Finance $(18.6 \%)$, Sales \& Marketing (18.4\%) and Operations (17.7\%) are approximately equal. It is analysed that most of the respondents are from MNL (37.7 \%), although there are approximately same number of respondents, i.e. the employees from UML (31.8 \%) and ML (30.4 \%).
While analysing the tenure of the respondents in their present organisations, it is found that most of the respondents are having less than 1 year of experience (30.4\%), 1-2 year of experience (32.5\%) and 2-5 years of experience (24.1\%) and very less respondents are working for more than 5 years $(13 \%)$. Other employees are It is clear that only $5.2 \%$ of the respondents are the employees with less than 1 year of total experience, $8.5 \%$ are employees with 1-2 years of total experience and $23.1 \%$ are employees with 2-5 years of total experience (in IT sector); and the sample is representative of the employees whose total experience in IT sector is more than 5 years $(63.2 \%)$, irrespective of the organisations they have worked.

\section{Data Collection Instrument}

The reliability of the various constructs of the questionnaire has been measured by using Cronbach's alpha. The greater the Cronbach's alpha, higher is the correlation amid the items and variables. Based on this, the reliability of the instrument is confirmed as all values are above 0.70 .

Table 1: Reliability analysis of the constructs

\begin{tabular}{|l|l|}
\hline Constructs & Cronbach' alpha \\
\hline Explicit Knowledge Sharing (EKS) & 0.904 \\
\hline Tacit Knowledge Sharing (TKS) & 0.917 \\
\hline Organisation related Tacit Knowledge Sharing (OTKS) & 0.876 \\
\hline Project/task related Tacit Knowledge Sharing (PTKS) & 0.859 \\
\hline Skill related Tacit Knowledge Sharing (STKS) & 0.842 \\
\hline Organisation related Explicit Knowledge Sharing (OEKS) & 0.868 \\
\hline Project/task related Explicit Knowledge Sharing (PEKS) & 0.846 \\
\hline Skill related Explicit Knowledge Sharing (SEKS) & 0.837 \\
\hline Customer metric organizational performance (COP) & 0.953 \\
\hline Process metric organizational performance (POP) & 0.917 \\
\hline People metric organizational performance (PLOP) & 0.943 \\
\hline Financial metric organizational performance (FOP) & 0.942 \\
\hline
\end{tabular}




\section{DATA ANALYSIS}

\section{Confirmatory Factor Analysis (CFA)}

CFA model of Knowledge sharing and Organizational performance are shown in Figure 2 and 3 respectively and their results are shown in Table 2 and 3 respectively.

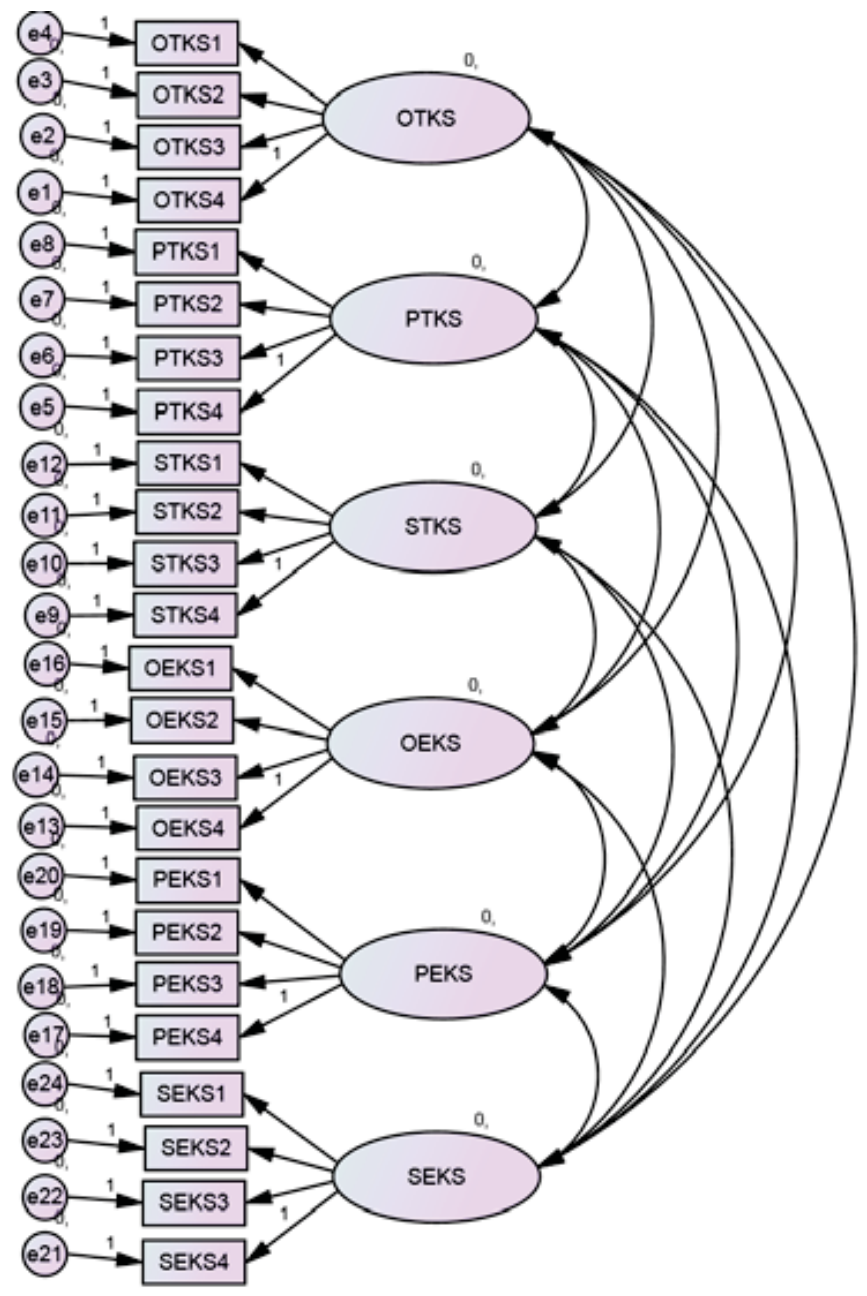

Figure 2: CFA model for Knowledge Sharing

Table 2: Results of Convergent and Discriminant Validity of Knowledge sharing CFA model

\begin{tabular}{|c|c|c|c|c|c|c|c|c|c|c|}
\hline & CR & AVE & MSV & ASV & SEKS & OTKS & PTKS & STKS & OEKS & PEKS \\
\hline SEKS & 0.916 & 0.731 & 0.579 & 0.243 & 0.855 & & & & & \\
\hline OTKS & 0.911 & 0.721 & 0.223 & 0.141 & 0.238 & 0.849 & & & & \\
\hline PTKS & 0.918 & 0.736 & 0.441 & 0.180 & 0.313 & 0.472 & 0.858 & & & \\
\hline STKS & 0.914 & 0.727 & 0.441 & 0.242 & 0.445 & 0.442 & 0.664 & 0.853 & & \\
\hline OEKS & 0.924 & 0.752 & 0.004 & 0.327 & 0.532 & 0.339 & 0.258 & 0.410 & 0.867 & \\
\hline PEKS & 0.815 & 0.532 & 0.004 & 0.396 & 0.761 & 0.343 & 0.268 & 0.457 & 1.002 & 0.729 \\
\hline
\end{tabular}




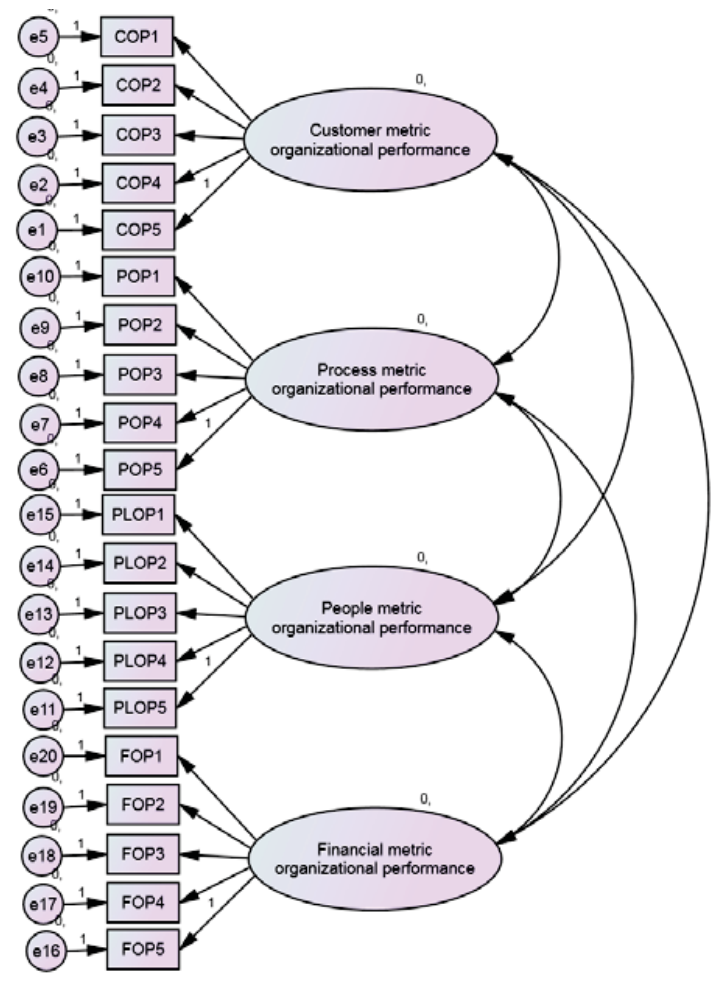

Figure 3: CFA model for Organizational Performance

Table 3: Results of Convergent and Discriminant Validity for organizational performance CFA model

\begin{tabular}{|c|c|c|c|c|c|c|c|c|}
\hline & CR & AVE & MSV & ASV & FOP & COP & POP & PLOP \\
\hline FOP & 0.861 & 0.614 & 0.055 & 0.019 & 0.784 & & & \\
\hline COP & 0.943 & 0.768 & 0.133 & 0.046 & 0.034 & 0.877 & & \\
\hline POP & 0.895 & 0.646 & 0.133 & 0.066 & 0.234 & 0.365 & 0.804 & \\
\hline PLOP & 0.708 & 0.514 & 0.009 & 0.004 & 0.031 & -0.050 & 0.093 & 0.643 \\
\hline
\end{tabular}

The results indicate that all Composite Reliability (CR) of both the models are more than 0.7, Average Variance Extracted (AVE) is more 0.5, and also CR is more than AVE hence, there are no convergent validity issues. Also for both the models, all Maximum Shared Variance (MSV) are more than AVE and Average Shared Variance (ASV) is more than AVE. Therefore, there are no discriminant validity issues. This indicates that the six-factor knowledge sharing model and four factors organizational performance model fitted the data reasonably well.

\section{Structure Equation Modelling}

To analyse various hypotheses stated above SEM was employed. Structural equation modelling (SEM), which is also referred to as covariance structure analysis, was used to examine the relationship amid tacit and explicit knowledge sharing and organizational performance. In the structural model, the study inspects absolute (e.g., goodness-of- fit index) in addition to incremental (e.g., comparative fit index, CFI) path coefficients and model fit indices (Byrne 2001). In gist, structural model simultaneously studies the numerous associations among the variables in a model, and anticipates measurement errors (Byrne 2001; Hayduk 1987). 


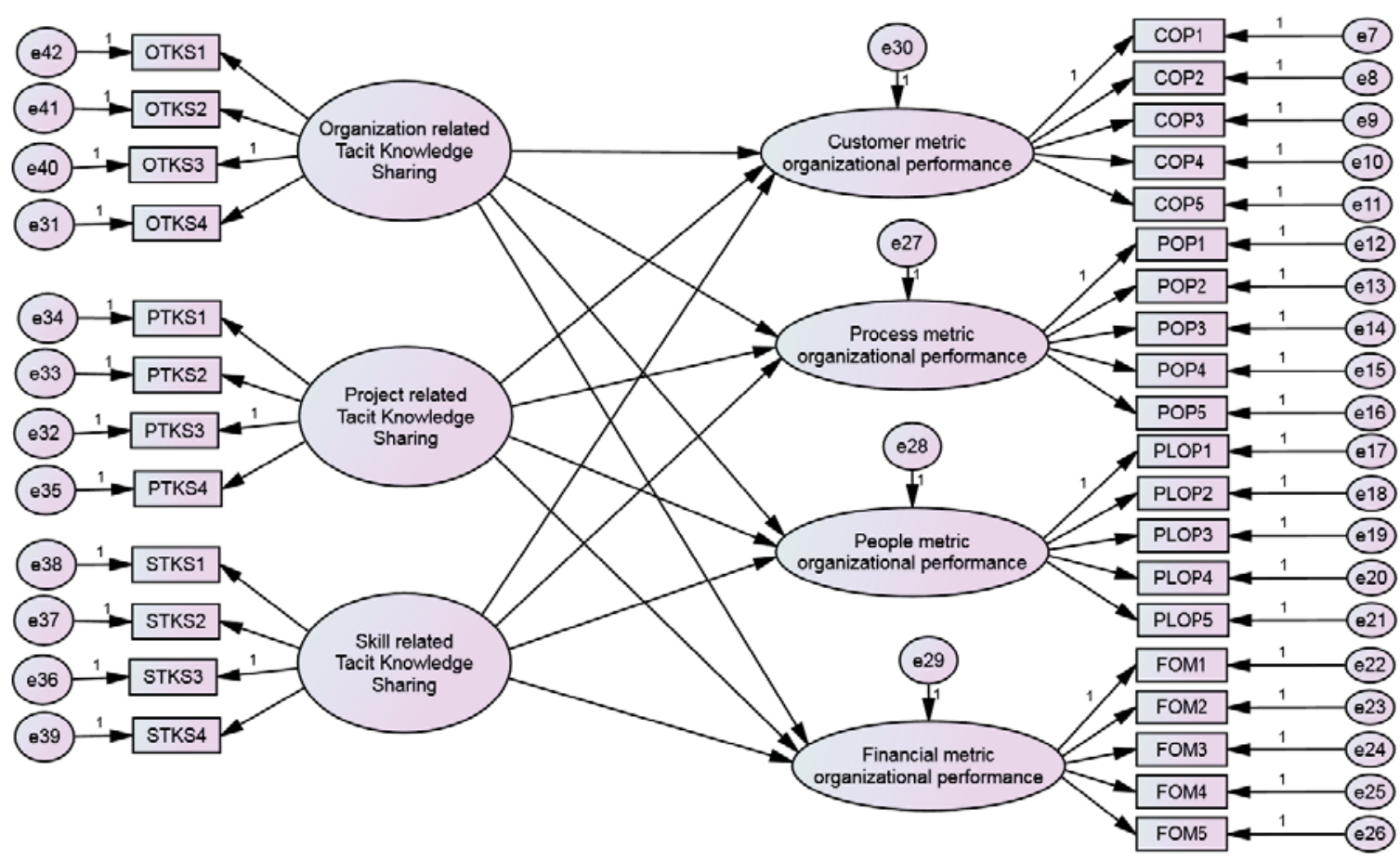

Figure 4: Structural Equation Model of "Impact of Tacit Knowledge Sharing on various dimensions of Organizational Performance".

Table 4: Results of Structural Equation Model of "Impact of Tacit Knowledge Sharing on various dimensions of Organizational Performance".

\begin{tabular}{|c|c|c|c|c|c|c|}
\hline $\begin{array}{l}\text { Exogenous } \\
\text { Construct }\end{array}$ & $\begin{array}{c}\text { Endogenous } \\
\text { Construct }\end{array}$ & $\begin{array}{c}\text { Standardized } \\
\text { Regression } \\
\text { Coefficient }\end{array}$ & $\begin{array}{c}\text { Unstandardized } \\
\text { Regression } \\
\text { Coefficient }\end{array}$ & $C R$ & $\begin{array}{c}P \\
\text { Value }\end{array}$ & $\begin{array}{c}\text { Squared } \\
\text { Correlation }\end{array}$ \\
\hline $\begin{array}{l}\text { Organization } \\
\text { related Tacit } \\
\text { Knowledge } \\
\text { Sharing }\end{array}$ & $\begin{array}{l}\text { Customer } \\
\text { metric } \\
\text { organizational } \\
\text { performance }\end{array}$ & 0.278 & 0.269 & 4.827 & 0.008 & 0.122 \\
\hline $\begin{array}{l}\text { Organization } \\
\text { related Tacit } \\
\text { Knowledge } \\
\text { Sharing }\end{array}$ & $\begin{array}{l}\text { Process metric } \\
\text { organizational } \\
\text { performance }\end{array}$ & 0.219 & 0.301 & 2.168 & 0.081 & 0.183 \\
\hline $\begin{array}{l}\text { Organization } \\
\text { related Tacit } \\
\text { Knowledge } \\
\text { Sharing }\end{array}$ & $\begin{array}{l}\text { People metric } \\
\text { organizational } \\
\text { performance }\end{array}$ & 0.173 & 0.189 & 2.678 & 0.000 & 0.108 \\
\hline $\begin{array}{l}\text { Organization } \\
\text { related Tacit } \\
\text { Knowledge } \\
\text { Sharing }\end{array}$ & $\begin{array}{l}\text { Financial } \\
\text { metric } \\
\text { organizational } \\
\text { performance }\end{array}$ & 0.278 & 0.269 & 3.857 & 0.060 & 0.165 \\
\hline
\end{tabular}




\begin{tabular}{|c|c|c|c|c|c|c|}
\hline $\begin{array}{l}\text { Project related } \\
\text { Tacit } \\
\text { Knowledge } \\
\text { Sharing }\end{array}$ & $\begin{array}{l}\text { Customer } \\
\text { metric } \\
\text { organizational } \\
\text { performance }\end{array}$ & 0.291 & 0.301 & 1.168 & 0.070 & 0.122 \\
\hline $\begin{array}{l}\text { Project related } \\
\text { Tacit } \\
\text { Knowledge } \\
\text { Sharing }\end{array}$ & $\begin{array}{l}\text { Process metric } \\
\text { organizational } \\
\text { performance }\end{array}$ & 0.191 & 0.189 & 2.678 & 0.003 & 0.183 \\
\hline $\begin{array}{l}\text { Project related } \\
\text { Tacit } \\
\text { Knowledge } \\
\text { Sharing }\end{array}$ & $\begin{array}{l}\text { People metric } \\
\text { organizational } \\
\text { performance }\end{array}$ & 0.291 & 0.176 & 1.462 & 0.001 & 0.108 \\
\hline $\begin{array}{l}\text { Project related } \\
\text { Tacit } \\
\text { Knowledge } \\
\text { Sharing }\end{array}$ & $\begin{array}{l}\text { Financial } \\
\text { metric } \\
\text { organizational } \\
\text { performance }\end{array}$ & 0.154 & 0.256 & 1.244 & 0.000 & 0.165 \\
\hline $\begin{array}{l}\text { Skill related } \\
\text { Tacit } \\
\text { Knowledge } \\
\text { Sharing }\end{array}$ & $\begin{array}{l}\text { Customer } \\
\text { metric } \\
\text { organizational } \\
\text { performance }\end{array}$ & 0.187 & 0.278 & 2.344 & 0.068 & 0.122 \\
\hline $\begin{array}{l}\text { Skill related } \\
\text { Tacit } \\
\text { Knowledge } \\
\text { Sharing }\end{array}$ & $\begin{array}{l}\text { Process metric } \\
\text { organizational } \\
\text { performance }\end{array}$ & 0.149 & 0.267 & 1.652 & 0.000 & 0.183 \\
\hline $\begin{array}{l}\text { Skill related } \\
\text { Tacit } \\
\text { Knowledge } \\
\text { Sharing }\end{array}$ & $\begin{array}{l}\text { People metric } \\
\text { organizational } \\
\text { performance }\end{array}$ & 0.158 & 0.149 & 1.943 & 0.000 & 0.108 \\
\hline $\begin{array}{l}\text { Skill related } \\
\text { Tacit } \\
\text { Knowledge } \\
\text { Sharing }\end{array}$ & $\begin{array}{l}\text { Financial } \\
\text { metric } \\
\text { organizational } \\
\text { performance }\end{array}$ & 0.231 & 0.258 & 2.769 & 0.000 & 0.165 \\
\hline
\end{tabular}

Table 5: Goodness of Fit Indices of Structural Equation Model of "Impact of Tacit Knowledge Sharing on various dimensions of Organizational Performance"

\begin{tabular}{|c|c|c|c|c|c|c|}
\hline $\begin{array}{c}\text { Fitness } \\
\text { Index }\end{array}$ & CFI & NFI & RFI & RMSEA & LO 90 & HI 90 \\
\hline Value & .928 & .907 & .849 & .115 & .156 & .175 \\
\hline
\end{tabular}

The outcomes of the investigation are presented in Table 4. The consequences specify that the probability value of the influence of the Organization related Tacit knowledge sharing on Customer metric and Process metric organizational performance is less than $5 \%$. Therefore, the null hypothesis of no influence of the construct Organization related Tacit knowledge sharing on Customer metric and Process metric organizational performance cannot be accepted. Correspondingly, the probability value of the influence of the Project related Tacit knowledge sharing and Skill related Tacit knowledge sharing on People metric, Financial metric and Process metric organizational performance is less than $5 \%$. Consequently, it can be determined that there subsists substantial influence of Project related Tacit knowledge sharing and Skill related Tacit knowledge sharing on People metric, 
Financial metric and Process metric organizational performance in IT organizations in India. CFI (0.928), NFI (0.907) and RFI (0.849) are high demonstrating that the structural model is appropriate. The multiple squared correlations of $12.2 \%, 18.3 \%, 10.8$ $\%$ and $16.5 \%$ signifies that $12.2 \%, 18.3 \%, 10.8 \%$ and $16.5 \%$ of the variations of the endogenous constructs Customer metric, Process metric, People metric, and Financial metric organizational performance respectively can be elucidated with the assistance of the deviations in the exogenous constructs Organization, process and skill related Tacit knowledge sharing.

Now, structural model of the impact of Explicit Knowledge Sharing on various dimensions of Organizational Performance is developed.

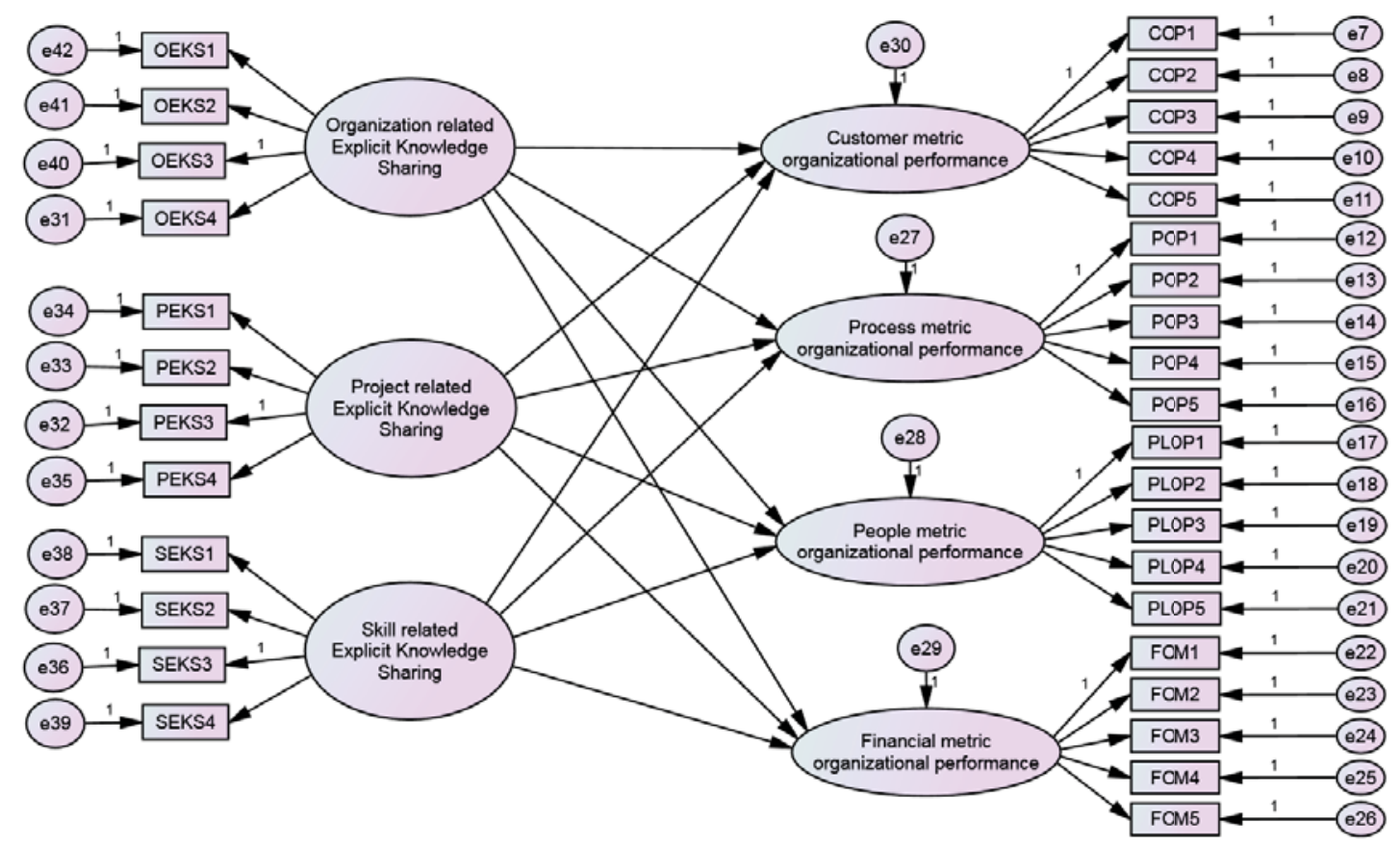

Figure 5: Structural Equation Model of "Impact of Explicit Knowledge Sharing on various dimensions of Organizational Performance".

Table 6: Results of Structural Equation Model of "Impact of Explicit Knowledge Sharing on various dimensions of Organizational Performance".

\begin{tabular}{|l|l|c|c|c|c|c|}
\hline \multicolumn{1}{|c|}{ Exogenous } & \multicolumn{1}{|c|}{$\begin{array}{c}\text { Endogenous } \\
\text { Construct }\end{array}$} & $\begin{array}{c}\text { Standardized } \\
\text { Regression } \\
\text { Coefficient }\end{array}$ & $\begin{array}{c}\text { Unstandardized } \\
\text { Regression } \\
\text { Coefficient }\end{array}$ & CR & $\begin{array}{c}P \\
\text { Value }\end{array}$ & $\begin{array}{c}\text { Squared } \\
\text { Correlation }\end{array}$ \\
\hline $\begin{array}{l}\text { Organization } \\
\text { related Explicit } \\
\text { Knowledge } \\
\text { Sharing }\end{array}$ & $\begin{array}{l}\text { Customer } \\
\text { metric } \\
\text { organizational } \\
\text { performance }\end{array}$ & 0.187 & 0.198 & 2.782 & 0.000 & 0.165 \\
\hline $\begin{array}{l}\text { Organization } \\
\text { related Explicit } \\
\text { Knowledge } \\
\text { Sharing }\end{array}$ & $\begin{array}{l}\text { Process metric } \\
\text { organizational } \\
\text { performance }\end{array}$ & 0.265 & 0.167 & 1.158 & 0.064 & 0.124 \\
\hline
\end{tabular}




\begin{tabular}{|c|c|c|c|c|c|c|}
\hline $\begin{array}{l}\text { Organization } \\
\text { related Explicit } \\
\text { Knowledge } \\
\text { Sharing }\end{array}$ & $\begin{array}{l}\text { People metric } \\
\text { organizational } \\
\text { performance }\end{array}$ & 0.237 & 0.198 & 4.346 & 0.000 & 0.180 \\
\hline $\begin{array}{l}\text { Organization } \\
\text { related Explicit } \\
\text { Knowledge } \\
\text { Sharing }\end{array}$ & $\begin{array}{l}\text { Financial } \\
\text { metric } \\
\text { organizational } \\
\text { performance }\end{array}$ & 0.167 & 0.196 & 2.763 & 0.080 & 0.121 \\
\hline $\begin{array}{l}\text { Project related } \\
\text { Explicit } \\
\text { Knowledge } \\
\text { Sharing }\end{array}$ & $\begin{array}{l}\text { Customer } \\
\text { metric } \\
\text { organizational } \\
\text { performance }\end{array}$ & 0.209 & 0.270 & 2.861 & 0.070 & 0.165 \\
\hline $\begin{array}{l}\text { Project related } \\
\text { Explicit } \\
\text { Knowledge } \\
\text { Sharing }\end{array}$ & $\begin{array}{l}\text { Process metric } \\
\text { organizational } \\
\text { performance }\end{array}$ & 0.154 & 0.159 & 3.768 & 0.003 & 0.124 \\
\hline $\begin{array}{l}\text { Project related } \\
\text { Explicit } \\
\text { Knowledge } \\
\text { Sharing }\end{array}$ & $\begin{array}{l}\text { People metric } \\
\text { organizational } \\
\text { performance }\end{array}$ & 0.195 & 0.267 & 2.638 & 0.091 & 0.180 \\
\hline $\begin{array}{l}\text { Project related } \\
\text { Explicit } \\
\text { Knowledge } \\
\text { Sharing }\end{array}$ & $\begin{array}{l}\text { Financial } \\
\text { metric } \\
\text { organizational } \\
\text { performance }\end{array}$ & 0.245 & 0.165 & 3.435 & 0.060 & 0.121 \\
\hline $\begin{array}{l}\text { Skill related } \\
\text { Explicit } \\
\text { Knowledge } \\
\text { Sharing }\end{array}$ & $\begin{array}{l}\text { Customer } \\
\text { metric } \\
\text { organizational } \\
\text { performance }\end{array}$ & 0.278 & 0.187 & 1.563 & 0.068 & 0.165 \\
\hline $\begin{array}{l}\text { Skill related } \\
\text { Explicit } \\
\text { Knowledge } \\
\text { Sharing } \\
\end{array}$ & $\begin{array}{l}\text { Process metric } \\
\text { organizational } \\
\text { performance }\end{array}$ & 0.298 & 0.176 & 1.874 & 0.082 & 0.124 \\
\hline $\begin{array}{l}\text { Skill related } \\
\text { Explicit } \\
\text { Knowledge } \\
\text { Sharing }\end{array}$ & $\begin{array}{l}\text { People metric } \\
\text { organizational } \\
\text { performance }\end{array}$ & 0.179 & 0.294 & 1.902 & 0.000 & 0.180 \\
\hline $\begin{array}{l}\text { Skill related } \\
\text { Explicit } \\
\text { Knowledge } \\
\text { Sharing }\end{array}$ & $\begin{array}{l}\text { Financial } \\
\text { metric } \\
\text { organizational } \\
\text { performance }\end{array}$ & 0.152 & 0.254 & 3.202 & 0.000 & 0.121 \\
\hline
\end{tabular}

Table 7: Goodness of Fit Indices of Structural Equation Model of "Impact of Explicit Knowledge Sharing on various dimensions of Organizational Performance".

\begin{tabular}{|c|c|c|c|c|c|c|}
\hline $\begin{array}{c}\text { Fitness } \\
\text { Index }\end{array}$ & CFI & NFI & RFI & RMSEA & LO 90 & HI 90 \\
\hline Value & .919 & .956 & .821 & .107 & .056 & .055 \\
\hline
\end{tabular}


The consequences of the investigation are presented in Table 6. The outcomes specify that the probability value of the influence of the Organization related Explicit knowledge sharing on Process metric and Financial metric organizational performance is more than $5 \%$. Therefore the null hypothesis of no influence of the construct Organization related Explicit knowledge sharing on Process metric and Financial metric organizational performance is accepted. Likewise, the probability value of the influence of the Project related Explicit knowledge sharing on Process metric organizational performance is less than $5 \%$. Consequently, it can be determined that there subsists substantial influence of Project related Explicit knowledge sharing on Process metric organizational performance in IT organizations in India. Also the probability value of the influence of the Skill related Explicit knowledge sharing on People metric and Financial metric organizational performance is less than $5 \%$ demonstrating there subsists substantial influence of Skill related Explicit knowledge sharing on People metric and Financial metric organizational performance. CFI (0.919), NFI (0.956) and RFI (0.821) are high demonstrating that the structural model is appropriate. The multiple squared correlations of $16.5 \%, 12.4 \%, 18 \%$ and $12.1 \%$ represents that 16.5 $\%, 12.4 \%, 18 \%$ and $12.1 \%$ of the variations of the endogenous constructs Customer metric, Process metric, People metric, and Financial metric organizational performance respectively can be explained with the help of the variations in the exogenous constructs Organization, process and skill related Explicit knowledge sharing.

3. In depth analysis of "Impact of Tacit and Explicit knowledge sharing on organizational performance"

To study the type of organization, process or skill related tacit and explicit knowledge that have more influence on organization performance of IT organisations in India, bivariate regression analysis is used.

Table 8: Bivariate Regression Model for Organization related Tacit Knowledge Sharing

\begin{tabular}{|c|c|c|c|c|c|c|}
\hline $\begin{array}{l}\text { Dependent } \\
\text { Variable }\end{array}$ & Independent Variable & $\begin{array}{c}\text { Beta } \\
\text { Coeffici } \\
\text { ent } \\
\end{array}$ & $\begin{array}{c}\text { Standar } \\
\text { dised } \\
\text { beta }\end{array}$ & $\begin{array}{l}t \text { statistic } \\
\text { ( } p \text { value) }\end{array}$ & $\begin{array}{l}\text { F statistic } \\
\text { ( } p \text { value) }\end{array}$ & $\mathbf{R}^{2}$ \\
\hline \multirow{4}{*}{$\begin{array}{l}\text { Customer } \\
\text { metric } \\
\text { organizational } \\
\text { performance }\end{array}$} & $\begin{array}{l}\text { Employee's business knowledge/ } \\
\text { non-financial data }\end{array}$ & 0.173 & 0.258 & $\begin{array}{l}5.480 \\
(.000)\end{array}$ & $\begin{array}{r}30.033 \\
(.000)\end{array}$ & $\begin{array}{c}0.06 \\
6\end{array}$ \\
\hline & $\begin{array}{l}\text { Employee's factual knowledge (know- } \\
\text { what) about the organisation }\end{array}$ & 0.171 & 0.248 & $\begin{array}{l}5.268 \\
(.000)\end{array}$ & $\begin{array}{r}27.748 \\
(.000)\end{array}$ & $\begin{array}{c}0.06 \\
2\end{array}$ \\
\hline & $\begin{array}{l}\text { Employee's financial knowledge of } \\
\text { the organisation }\end{array}$ & 0.269 & 0.186 & $\begin{array}{l}5.740 \\
(.000)\end{array}$ & $\begin{array}{r}32.953 \\
(.000)\end{array}$ & $\begin{array}{c}0.07 \\
2\end{array}$ \\
\hline & $\begin{array}{l}\text { Employee's own acquaintances about } \\
\text { the organization }\end{array}$ & 0.300 & 0.207 & $\begin{array}{l}6.451 \\
(.000)\end{array}$ & $\begin{array}{l}41.611 \\
(.000)\end{array}$ & $\begin{array}{c}0.09 \\
0\end{array}$ \\
\hline \multirow{4}{*}{$\begin{array}{l}\text { People metric } \\
\text { organizational } \\
\text { performance }\end{array}$} & $\begin{array}{l}\text { Employee's business knowledge/ } \\
\text { non-financial data }\end{array}$ & 0.271 & 0.375 & $\begin{array}{l}8.318 \\
(.000)\end{array}$ & $\begin{array}{l}69.193 \\
(.000)\end{array}$ & $\begin{array}{c}0.14 \\
1\end{array}$ \\
\hline & $\begin{array}{l}\text { Employee's factual knowledge (know- } \\
\text { what) about the organisation }\end{array}$ & 0.288 & 0.399 & $\begin{array}{l}8.934 \\
(.000)\end{array}$ & $\begin{array}{c}79.824 \\
(.000)\end{array}$ & $\begin{array}{c}0.15 \\
9\end{array}$ \\
\hline & $\begin{array}{l}\text { Employee's financial knowledge of } \\
\text { the organisation }\end{array}$ & 0.332 & 0.376 & $\begin{array}{l}8.346 \\
(.000)\end{array}$ & $\begin{array}{r}69.660 \\
(.000)\end{array}$ & $\begin{array}{c}0.14 \\
2\end{array}$ \\
\hline & $\begin{array}{l}\text { Employee's own acquaintances about } \\
\text { the organization }\end{array}$ & 0.344 & 0.412 & $\begin{array}{l}9.292 \\
(.000)\end{array}$ & $\begin{array}{r}86.337 \\
(.000)\end{array}$ & $\begin{array}{c}0.17 \\
0\end{array}$ \\
\hline
\end{tabular}


Table 9: Bivariate Regression Model for Project related Tacit Knowledge Sharing

\begin{tabular}{|c|c|c|c|c|c|c|}
\hline $\begin{array}{l}\text { Dependent } \\
\text { Variable }\end{array}$ & Independent Variable & $\begin{array}{c}\text { Beta } \\
\text { Coefficient }\end{array}$ & $\begin{array}{c}\text { Standar } \\
\text { dised } \\
\text { beta }\end{array}$ & $\begin{array}{l}t \text { statistic } \\
\text { ( } p \text { value) }\end{array}$ & $\begin{array}{l}\text { F statistic } \\
\text { (p value) }\end{array}$ & $\mathbf{R}^{2}$ \\
\hline \multirow[t]{4}{*}{$\begin{array}{l}\text { Process metric } \\
\text { organizational } \\
\text { performance }\end{array}$} & $\begin{array}{l}\text { Employee's factual knowledge } \\
\text { (know-what) of the particular } \\
\text { project/task }\end{array}$ & 0.412 & 0.480 & $\begin{array}{c}11.248 \\
(.000)\end{array}$ & $\begin{array}{c}126.525 \\
(.000)\end{array}$ & $\begin{array}{c}0.23 \\
1\end{array}$ \\
\hline & $\begin{array}{l}\text { Employee's Know-how (how to do } \\
\text { particular task/project) knowledge } \\
\text { or tricks of the trade of the } \\
\text { particular project/task }\end{array}$ & 0.343 & 0.543 & $\begin{array}{l}11.943 \\
(.000)\end{array}$ & $\begin{array}{c}142.640 \\
(.000)\end{array}$ & $\begin{array}{c}0.25 \\
3\end{array}$ \\
\hline & $\begin{array}{l}\text { Employee's Know-why (why to do } \\
\text { particular task/project) knowledge }\end{array}$ & 0.420 & 0.511 & $\begin{array}{c}13.212 \\
(.000)\end{array}$ & $\begin{array}{c}174.547 \\
(.000)\end{array}$ & $\begin{array}{c}0.29 \\
3\end{array}$ \\
\hline & $\begin{array}{l}\text { Employee's own acquaintance of } \\
\text { the particular project/task }\end{array}$ & 0.338 & 0.478 & $\begin{array}{c}11.172 \\
(.000)\end{array}$ & $\begin{array}{c}124.808 \\
(.000)\end{array}$ & $\begin{array}{c}0.22 \\
8\end{array}$ \\
\hline \multirow{4}{*}{$\begin{array}{l}\text { People metric } \\
\text { organizational } \\
\text { performance }\end{array}$} & $\begin{array}{l}\text { Employee's factual knowledge } \\
\text { (know-what) of the particular } \\
\text { project/task }\end{array}$ & 0.360 & 0.470 & $\begin{array}{l}10.930 \\
(.000)\end{array}$ & $\begin{array}{c}119.454 \\
(.000)\end{array}$ & $\begin{array}{c}0.22 \\
1\end{array}$ \\
\hline & $\begin{array}{l}\text { Employee's Know-how (how to do } \\
\text { particular task/project) knowledge } \\
\text { or tricks of the trade of the } \\
\text { particular project/task }\end{array}$ & 0.344 & 0.466 & $\begin{array}{l}10.815 \\
(.000)\end{array}$ & $\begin{array}{c}116.964 \\
(.000)\end{array}$ & $\begin{array}{c}0.21 \\
7\end{array}$ \\
\hline & $\begin{array}{l}\text { Employee's Know-why (why to do } \\
\text { particular task/project) knowledge }\end{array}$ & 0.415 & 0.465 & $\begin{array}{c}10.786 \\
(.000)\end{array}$ & $\begin{array}{c}116.330 \\
(.000)\end{array}$ & $\begin{array}{c}0.21 \\
6\end{array}$ \\
\hline & $\begin{array}{l}\text { Employee's own acquaintance of } \\
\text { the particular project/task }\end{array}$ & 0.379 & 0.532 & $\begin{array}{c}12.912 \\
(.000)\end{array}$ & $\begin{array}{c}166.722 \\
(.000)\end{array}$ & $\begin{array}{c}0.28 \\
3\end{array}$ \\
\hline \multirow{4}{*}{$\begin{array}{c}\text { Financial } \\
\text { metric } \\
\text { organizational } \\
\text { performance }\end{array}$} & $\begin{array}{l}\text { Employee's factual knowledge } \\
\text { (know-what) of the particular } \\
\text { project/task }\end{array}$ & 0.446 & 0.551 & $\begin{array}{c}13.565 \\
(.000)\end{array}$ & $\begin{array}{c}184.015 \\
(.000)\end{array}$ & $\begin{array}{c}0.30 \\
4\end{array}$ \\
\hline & $\begin{array}{l}\text { Employee's Know-how (how to do } \\
\text { particular task/project) knowledge } \\
\text { or tricks of the trade of the } \\
\text { particular project/task }\end{array}$ & 0.355 & 0.482 & $\begin{array}{c}11.293 \\
(.000)\end{array}$ & $\begin{array}{c}127.524 \\
(.000)\end{array}$ & $\begin{array}{c}0.23 \\
2\end{array}$ \\
\hline & $\begin{array}{l}\text { Employee's Know-why (why to do } \\
\text { particular task/project) knowledge }\end{array}$ & 0.369 & 0.462 & $\begin{array}{c}10.700 \\
(.000)\end{array}$ & $\begin{array}{c}114.489 \\
(.000)\end{array}$ & $\begin{array}{c}0.21 \\
3\end{array}$ \\
\hline & $\begin{array}{l}\text { Employee's own acquaintance of } \\
\text { the particular project/task }\end{array}$ & 0.362 & 0.471 & $\begin{array}{c}10.956 \\
(.000)\end{array}$ & $\begin{array}{c}120.035 \\
(.000)\end{array}$ & $\begin{array}{c}0.22 \\
1\end{array}$ \\
\hline
\end{tabular}


Table10: Bivariate Regression Model for Skill related Tacit Knowledge Sharing

\begin{tabular}{|c|c|c|c|c|c|c|}
\hline $\begin{array}{l}\text { Dependent } \\
\text { Variable }\end{array}$ & Independent Variable & $\begin{array}{c}\text { Beta } \\
\text { Coeffici } \\
\text { ent }\end{array}$ & $\begin{array}{c}\text { Standar } \\
\text { dised } \\
\text { beta }\end{array}$ & $\begin{array}{l}t \text { statistic } \\
\text { ( } p \text { value) }\end{array}$ & $\begin{array}{l}\text { F statistic } \\
\text { ( } p \text { value) }\end{array}$ & $\mathbf{R}^{2}$ \\
\hline \multirow{5}{*}{$\begin{array}{l}\text { Process metric } \\
\text { organizational } \\
\text { performance }\end{array}$} & $\begin{array}{l}\text { Employee's experiences or soft skills } \\
\text { related knowledge }\end{array}$ & 0.248 & 0.329 & $\begin{array}{l}7.159 \\
(.000)\end{array}$ & $\begin{array}{l}51.247 \\
(.000)\end{array}$ & $\begin{array}{c}0.10 \\
8\end{array}$ \\
\hline & $\begin{array}{l}\text { Employee's analytical or problem } \\
\text { solving skills }\end{array}$ & 0.298 & 0.374 & $\begin{array}{l}8.037 \\
(.000)\end{array}$ & $\begin{array}{c}64.592 \\
(.000)\end{array}$ & $\begin{array}{c}0.13 \\
3\end{array}$ \\
\hline & Employee's technological expertise & 0.297 & 0.363 & $\begin{array}{l}8.009 \\
(.000) \\
\end{array}$ & $\begin{array}{r}64.139 \\
(.000) \\
\end{array}$ & $\begin{array}{c}0.13 \\
2 \\
\end{array}$ \\
\hline & Employee's job-related hard skills & 0.281 & 0.370 & $\begin{array}{l}8.177 \\
(.000)\end{array}$ & $\begin{array}{c}66.856 \\
(.000)\end{array}$ & $\begin{array}{c}0.13 \\
7\end{array}$ \\
\hline & $\begin{array}{l}\text { Employee's experiences or soft skills } \\
\text { related knowledge }\end{array}$ & 0.305 & 0.408 & $\begin{array}{l}9.177 \\
(.000)\end{array}$ & $\begin{array}{c}84.220 \\
(.000) \\
\end{array}$ & $\begin{array}{c}0.16 \\
6\end{array}$ \\
\hline \multirow{3}{*}{$\begin{array}{l}\text { People metric } \\
\text { organizational } \\
\text { performance }\end{array}$} & $\begin{array}{l}\text { Employee's analytical or problem } \\
\text { solving skills }\end{array}$ & 0.273 & 0.344 & $\begin{array}{l}7.538 \\
(.000) \\
\end{array}$ & $\begin{array}{c}56.815 \\
(.000) \\
\end{array}$ & $\begin{array}{c}0.11 \\
9\end{array}$ \\
\hline & Employee's technological expertise & 0.332 & 0.435 & $\begin{array}{l}9.917 \\
(.000)\end{array}$ & $\begin{array}{c}98.352 \\
(.000)\end{array}$ & $\begin{array}{c}0.18 \\
9\end{array}$ \\
\hline & Employee's job-related hard skills & 0.313 & 0.377 & $\begin{array}{l}8.363 \\
(.000) \\
\end{array}$ & $\begin{array}{r}69.937 \\
(.000) \\
\end{array}$ & $\begin{array}{c}0.14 \\
2 \\
\end{array}$ \\
\hline \multirow{4}{*}{$\begin{array}{c}\text { Financial } \\
\text { metric } \\
\text { organizational } \\
\text { performance }\end{array}$} & $\begin{array}{l}\text { Employee's experiences or soft skills } \\
\text { related knowledge }\end{array}$ & 0.305 & 0.357 & $\begin{array}{l}7.861 \\
(.000)\end{array}$ & $\begin{array}{c}61.802 \\
(.000)\end{array}$ & $\begin{array}{c}0.12 \\
8\end{array}$ \\
\hline & $\begin{array}{l}\text { Employee's analytical or problem } \\
\text { solving skills }\end{array}$ & 0.299 & 0.378 & $\begin{array}{l}8.393 \\
(.000)\end{array}$ & $\begin{array}{c}70.442 \\
(.000)\end{array}$ & $\begin{array}{c}0.14 \\
3\end{array}$ \\
\hline & Employee's technological expertise & 0.323 & 0.414 & $\begin{array}{l}9.335 \\
(.000) \\
\end{array}$ & $\begin{array}{c}87.138 \\
(.000) \\
\end{array}$ & $\begin{array}{c}0.17 \\
1 \\
\end{array}$ \\
\hline & Employee's job-related hard skills & 0.315 & 0.380 & $\begin{array}{l}8.451 \\
(.000)\end{array}$ & $\begin{array}{c}71.425 \\
(.000) \\
\end{array}$ & $\begin{array}{c}0.14 \\
5\end{array}$ \\
\hline
\end{tabular}

The results (Table 8, 9 and 10) of the regression analysis indicate that both all forms of tacit knowledge influence the four dimensions of organizational performance. The results clearly shows that if an organisation want to enhance Customer metric organizational performance, they should focus that employees should share their organization related business knowledge related to non-financial data $(\beta=0.258, \mathrm{t}=5.480)$ and if an organisation want to enhance Process metric organizational performance, they should focus that employees should share their Know-how (how to do particular task/project) knowledge or tricks of the trade of the particular project $/$ task $(\beta=0.543, \mathrm{t}=$
11.943) and analytical or problem solving skills $(\beta=$ $0.374, t=8.037)$. But if the focus of the organisation is to enhance People metric organizational performance, they should focus that employees should share their own acquaintances about the organization $(\beta=0.412, \mathrm{t}=9.292)$, their own acquaintance of the particular project/task $(\beta=$ $0.532, \mathrm{t}=12.912)$ and their technological expertise $(\beta$ $=0.435, \mathrm{t}=9.917)$. The results also suggest that if an organisation want to enhance Financial metric organizational performance, they should focus that employees should share their technological expertise $(\beta=0.435, \mathrm{t}=9.917)$. 
Table 11: Bivariate Regression Model for Organization related Explicit Knowledge Sharing

\begin{tabular}{|c|c|c|c|c|c|c|}
\hline $\begin{array}{l}\text { Dependent } \\
\text { Variable }\end{array}$ & Independent Variable & $\begin{array}{c}\text { Beta } \\
\text { Coefficient }\end{array}$ & $\begin{array}{l}\text { Standard } \\
\text { ised beta }\end{array}$ & $\begin{array}{l}t \text { statistic } \\
\text { ( } p \text { value) }\end{array}$ & $\begin{array}{l}\text { F statistic } \\
\text { ( } \mathrm{p} \text { value) }\end{array}$ & $\mathbf{R}^{2}$ \\
\hline \multirow{4}{*}{$\begin{array}{c}\text { Customer } \\
\text { metric } \\
\text { organizational } \\
\text { performance }\end{array}$} & $\begin{array}{l}\text { Documented business } \\
\text { knowledge/ non-financial data }\end{array}$ & 0.233 & 0.197 & $\begin{array}{l}4.918 \\
(.000)\end{array}$ & $\begin{array}{c}24.191 \\
(.000)\end{array}$ & 0.054 \\
\hline & $\begin{array}{l}\text { Documented factual knowledge } \\
\text { (know-what) about the } \\
\text { organisation }\end{array}$ & 0.238 & 0.298 & $\begin{array}{l}6.413 \\
(.000)\end{array}$ & $\begin{array}{l}41.126 \\
(.000)\end{array}$ & 0.089 \\
\hline & $\begin{array}{l}\text { Documented financial } \\
\text { knowledge of the organisation }\end{array}$ & 0.400 & 0.281 & $\begin{array}{l}8.978 \\
(.000)\end{array}$ & $\begin{array}{c}80.602 \\
(.000)\end{array}$ & 0.160 \\
\hline & $\begin{array}{l}\text { Documented acquaintances of } \\
\text { the employees about the } \\
\text { organization }\end{array}$ & 0.293 & 0.408 & $\begin{array}{l}9.176 \\
(.000)\end{array}$ & $\begin{array}{c}84.194 \\
(.000)\end{array}$ & 0.166 \\
\hline \multirow{4}{*}{$\begin{array}{l}\text { People metric } \\
\text { organizational } \\
\text { performance }\end{array}$} & $\begin{array}{l}\text { Documented business } \\
\text { knowledge/ non-financial data }\end{array}$ & 0.246 & 0.389 & $\begin{array}{l}8.677 \\
(.000)\end{array}$ & $\begin{array}{r}75.290 \\
(.000)\end{array}$ & 0.151 \\
\hline & $\begin{array}{l}\text { Documented factual knowledge } \\
\text { (know-what) about the } \\
\text { organisation }\end{array}$ & 0.242 & 0.385 & $\begin{array}{l}8.580 \\
(.000)\end{array}$ & $\begin{array}{l}73.608 \\
(.000)\end{array}$ & 0.149 \\
\hline & $\begin{array}{l}\text { Documented financial } \\
\text { knowledge of the organisation }\end{array}$ & 0.203 & 0.328 & $\begin{array}{l}7.140 \\
(.000)\end{array}$ & $\begin{array}{c}50.976 \\
(.000)\end{array}$ & 0.108 \\
\hline & $\begin{array}{l}\text { Documented acquaintances of } \\
\text { the employees about the } \\
\text { organization }\end{array}$ & 0.243 & 0.373 & $\begin{array}{l}8.252 \\
(.000)\end{array}$ & $\begin{array}{l}68.099 \\
(.000)\end{array}$ & 0.139 \\
\hline
\end{tabular}

Table 12: Bivariate Regression Model for Project related Explicit Knowledge Sharing

\begin{tabular}{|c|c|c|c|c|c|c|}
\hline $\begin{array}{l}\text { Dependent } \\
\text { Variable }\end{array}$ & Independent Variable & $\begin{array}{c}\text { Beta } \\
\text { Coefficient }\end{array}$ & $\begin{array}{l}\text { Standard } \\
\text { ised beta }\end{array}$ & $\begin{array}{l}\mathbf{t} \text { statistic } \\
\text { ( } \mathrm{p} \text { value) }\end{array}$ & $\begin{array}{l}\text { F statistic } \\
\text { ( } \mathrm{p} \text { value) }\end{array}$ & $\mathbf{R}^{2}$ \\
\hline \multirow{4}{*}{$\begin{array}{c}\text { Process metric } \\
\text { organizational } \\
\text { performance }\end{array}$} & $\begin{array}{l}\text { Documented factual } \\
\text { knowledge (know-what) of the } \\
\text { particular project/task }\end{array}$ & 0.317 & 0.414 & $\begin{array}{l}9.344 \\
(.000)\end{array}$ & $\begin{array}{r}87.313 \\
(.000)\end{array}$ & 0.171 \\
\hline & $\begin{array}{l}\text { Documented Know-how (how } \\
\text { to do particular task/project) } \\
\text { knowledge or tricks of the } \\
\text { trade of the particular } \\
\text { project/task }\end{array}$ & 0.297 & 0.426 & $\begin{array}{l}9.684 \\
(.000)\end{array}$ & $\begin{array}{c}93.778 \\
(.000)\end{array}$ & 0.182 \\
\hline & $\begin{array}{l}\text { Documented Know-why (why } \\
\text { to do particular task/project) } \\
\text { knowledge }\end{array}$ & 0.344 & 0.455 & $\begin{array}{l}10.499 \\
(.000)\end{array}$ & $\begin{array}{c}110.227 \\
(.000)\end{array}$ & 0.207 \\
\hline & $\begin{array}{l}\text { Documented own acquaintance } \\
\text { of the particular project/task }\end{array}$ & 0.343 & 0.471 & $\begin{array}{r}10.965 \\
(.000)\end{array}$ & $\begin{array}{c}120.242 \\
(.000)\end{array}$ & 0.222 \\
\hline
\end{tabular}


Table13: Bivariate Regression Model for Skill related Tacit Knowledge Sharing

\begin{tabular}{|c|l|c|c|c|c|c|}
\hline $\begin{array}{c}\text { Dependent } \\
\text { Variable }\end{array}$ & \multicolumn{1}{|c|}{ Independent Variable } & $\begin{array}{c}\text { Beta } \\
\text { Coefficient }\end{array}$ & $\begin{array}{c}\text { Standard } \\
\text { ised beta }\end{array}$ & $\begin{array}{c}\text { t statistic } \\
\text { (p value) }\end{array}$ & $\begin{array}{c}\text { F statistic } \\
\text { (p value) }\end{array}$ & $\mathbf{R}^{2}$ \\
\hline \multirow{4}{*}{$\begin{array}{c}\text { People metric } \\
\text { organizational } \\
\text { performance }\end{array}$} & $\begin{array}{l}\text { Documented experiences or } \\
\text { soft skills related knowledge }\end{array}$ & 0.307 & 0.380 & $\begin{array}{c}8.443 \\
(.000)\end{array}$ & $\begin{array}{c}71.288 \\
(.000)\end{array}$ & 0.145 \\
\cline { 2 - 7 } & $\begin{array}{l}\text { Documented analytical or } \\
\text { problem solving skills }\end{array}$ & 0.262 & 0.350 & $\begin{array}{c}7.687 \\
(.000)\end{array}$ & $\begin{array}{c}59.092 \\
(.000)\end{array}$ & 0.123 \\
\cline { 2 - 7 } & $\begin{array}{l}\text { Documented technological } \\
\text { expertise }\end{array}$ & 0.240 & 0.325 & $\begin{array}{c}7.066 \\
(.000)\end{array}$ & $\begin{array}{c}49.933 \\
(.000)\end{array}$ & 0.106 \\
\cline { 2 - 7 } & $\begin{array}{l}\text { Documented job-related hard } \\
\text { skills }\end{array}$ & 0.229 & 0.292 & $\begin{array}{c}6.267 \\
(.000)\end{array}$ & $\begin{array}{c}39.278 \\
(.000)\end{array}$ & 0.085 \\
\hline \multirow{4}{*}{$\begin{array}{c}\text { Financial metric } \\
\text { organizational } \\
\text { performance }\end{array}$} & $\begin{array}{l}\text { Documented experiences or } \\
\text { soft skills related knowledge }\end{array}$ & 0.323 & 0.414 & $\begin{array}{c}9.335 \\
(.000)\end{array}$ & $\begin{array}{c}87.138 \\
(.000)\end{array}$ & 0.171 \\
\cline { 2 - 8 } & $\begin{array}{l}\text { Documented analytical or } \\
\text { problem solving skills }\end{array}$ & 0.315 & 0.380 & $\begin{array}{c}8.451 \\
(.000)\end{array}$ & $\begin{array}{c}71.425 \\
(.000)\end{array}$ & 0.145 \\
\cline { 2 - 8 } & Documented technological & 0.329 & 0.413 & $\begin{array}{c}9.329 \\
(.000)\end{array}$ & $\begin{array}{c}87.034 \\
(.000)\end{array}$ & 0.171 \\
\cline { 2 - 8 } & $\begin{array}{l}\text { Documented job-related hard } \\
\text { skills }\end{array}$ & 0.335 & 0.388 & $\begin{array}{c}8.651 \\
(.000)\end{array}$ & $\begin{array}{c}74.844 \\
(.000)\end{array}$ & 0.151 \\
\hline
\end{tabular}

The results (Table 11, 12 and 13) of the regression analysis indicate that both all forms of explicit knowledge influence the four dimensions of organizational performance. The results clearly shows that if an organisation want to enhance Customer metric organizational performance, they should focus that employees should share documented acquaintances of the employees about the organization $(\beta=0.408, \mathrm{t}=9.176)$ and if an organisation want to enhance Process metric organizational performance, they should focus that employees should share documented own acquaintance of the particular project/task $(\beta=$ $0.471, t=10.965)$. But if the focus of the organisation is to enhance People metric organizational performance, they should focus that employees should share documented business knowledge/ non-financial data $(\beta=0.389, \mathrm{t}=8.677)$, and documented experiences or soft skills related knowledge $(\beta=0.380, t=8.443)$. The results also suggest that if an organisation want to enhance Financial metric organizational performance, they should focus that employees should share
Documented experiences or soft skills related knowledge $(\beta=0.414, t=9.335)$

\section{CONCLUSION}

Various organizational processes, comparable to modernization, collective policymaking, discrete, and communal erudition are significantly impacted/improved by knowledge sharing. Chu (2000) in line with Jian (2007) empirically proves that the blend of knowledge amalgamation process and modernization approaches has an affirmative and substantial impact on the presentation of knowledge transformation. With the enhanced organizational progressions there is enhancement in organizational performance. Numerous research works deliberated the influence of administration of knowledge on enactment of organization; though, there is inadequate theory that efficaciously demarcated the variable "organizational performance" as an imperative mechanism for maintaining competitive edge (Sanchez and Mahoney 1996; Alvi and Leidner 2001; Gold et al. 2001; Lee and Choi, 2003; Acar and 
Acar, 2014; Ahmed and Shafiq, 2014; Turel and Bart, 2014).

In a developing country like India, where IT industry has huge scope for expansion as well as development and is predictable to propagate further, there is lot of competition. To gain this competitive edge the organization needs to encourage their employees for innovative products and services that can attract customers which in turn helps to attain and sustain competitive advantage (Bhatti, 2011). The research supports organizations not only to comprehend the important impact of tacit and Explicit knowledge sharing on organizational performance but will also help to scrutinize that which form of tacit and explicit knowledge will influence organizational performance more $n$ IT organizations in India.

\section{LIMITATIONS OF THE STUDY}

The foremost limitation of the study is that during analyzing the antecedent studies, it was found that limited literature is available on the theme. Also, although forms of tacit and Explicit knowledge and dimensions of organizational performance of IT companies in India, studied in the current research have been vetted by the experts of academics and industry, still there could be many parameters that have influence on organizational performance and are understudied. The other limitation is the static nature of the study. As data collection tool used is questionnaire, so response bias limitation cannot be overlooked.

\section{REFERENCES}

1) Acar, A. Z., \& Acar, P. (2014). Organizational culture types and their effects on organizational performance in Turkish hospitals. EMAJ: Emerging Markets Journal, 3(3), 18-31.

2) Ahmed, S. S. (2014). The impact of organizational culture on organizational performance: a case study on telecom sector. Global Journal of Management and Business
Research, 14(3), 20-30.

3) Al-Alawi A I, Al-Marzooqi N Y and Mohammed Y F (2007), "Organizational Culture and Knowledge Sharing: Critical Success Factors", Journal of Knowledge Management, Vol. 11, No. 2, pp. 22-42

4) Alavi, M., \& Leidner, D. E. (2001). Review: knowledge management and knowledge management systems: conceptual foundations and research issues. MIS quarterly, 107-136.

5) Argote, L. and Ingram, P. (2000), "Knowledge transfer: a basis for competitive advantage infirms", Organizational Behavior and Human Decision Processes, Vol. 8 No. 1, pp. 156-169.

6) Argote, L. and Ingram, P. (2000), “Knowledge transfer: a basis for competitive advantage in firms", Organizational Behavior and Human Decision Processes, Vol. 82 No. 1, pp. 150-169.

7) Berends H (2005), “Exploring Knowledge Sharing: Moves, Problem Solving and Justification", Knowledge Management Research \& Practice, Vol. 3, No. 2, pp. 97-105.

8) Boh, W.F. (2007), "Mechanisms for sharing knowledge in project-based organizations", Information and Organization, Vol. 17 No. 1, pp. 27-58.

9) Burke, M. (2011). Knowledge sharing in emerging economies. Library Review, 60(1), 514. doi:10.1108/00242531111100531.

10) Chen, C. J., Huang, J. W., \& Hsiao, Y. C. (2010). Knowledge management and innovativeness the role of organisational climate and structure. International Journal of Manpower, 31(8), 848870.

11) Cheng W, Hailin L and Hongming $X$ (2008), "Does Knowledge Sharing Mediate the Relationship Between Trust and Performance?", International Symposiums on Information Processing, IEEE.

12) Cheng, J. (2011), “Interorganizational relationships and knowledge sharing in green supply chains-Moderating by relational benefits and guanxi", Transportation Review, Part E: Logistics and Transportation Review, Vol. 47 No. 6, pp. 837-849. 
13) Chu, Pei-Ying (2000) A study of how the combination of knowledge integration mechanism and innovation strategies affects the knowledge conversion performance: a comparison between high-tech firms and service providers, MA, Department of Business Administration, Chung Yuan Christian University

14) Connelly C E and Kelloway E K (2003), "Predictors of Employees Perceptions of Knowledge Sharing Culture", Leadership and Organizational Development Journal, Vol. 24, No. 5, pp. 294-301

15) Covin J G and Slevin D P (1989), "Strategic Management of Small Firms in Hostile and Benign Environments", Strategic Management Journal, Vol. 10, No. 1, pp. 75-87.

16) Darroch J (2005), “Knowledge Management, Innovation and Firm Performance", Journal of Knowledge Management, Vol. 9, No. 3, pp. 101115.

17) Dess G G and Robinson R B (1984), “Measuring Organizational Performance in the Absence of Objective Measures: The Case of the PrivatelyHeld Firm and Conglomerate Business Unit", Strategic Management Journal, Vol. 5, No. 3, pp. 265-273.

18) Du R, Ai S and Ren Y (2007), "Relationship Between Knowledge Sharing and Performance: A Survey in Xi'an, China", Expert Systems with Applications, Vol. 32, No. 1, pp. 38-46.

19) Fey C F and Furu P (2008), “Top Management Incentive Compensation and Knowledge Sharing in Multinational Corporations", Strategic Management Journal, Vol. 29, No. 12, pp. 1301-1323.

20) Ford, D. P., \& Staples, S. (2010). Are full and partial Knowledge Sharing the same?. Journal of Knowledge Management, 14(3), 394-409.

21) Gold, A. H., Malhotra, A., \& Segars, A. H. (2001). Knowledge management: an organizational capabilities perspective. Journal of Management Information Systems, 18(1), 185-214.

22) Gupta S K (2008), “A Comparative Analysis of Knowledge Sharing Climate", Journal of
Knowledge and Process Management, Vol. 15, No. 3, pp. 186-195.

23) Hedlund G (1994), "A Model of Knowledge Management and the N-Form Corporation", Strategic Management Journal, Vol. 15, Summer, pp. 73-90.

24) Hoffman J J, Hoelscher M L and Sherif K (2005), "Social Capital, Knowledge Management and Sustained Superior Performance", Journal of Knowledge Management, Vol. 9, No. 3, pp. 93100.

25) Hsu C I and Wang S Y (2008), "A Model of IntraOrganizational Knowledge Sharing: Development and Initial Test", Journal of Global Information Management, Vol. 16, No. 3, pp. 4573.

26) Hsu I C (2008), “Knowledge Sharing Practices as a Facilitating Factor for Improving Organizational Performance Through Human Capital: A Preliminary Test", Expert Systems with Applications, Vol. 35, No. 3, pp. 1316-1326.

27) Ipe, M. (2003), "Knowledge sharing in organization: a conceptual framework", Human Resource Development Review, Vol. 2 No. 4, pp. 337-359.

28) Jian, Y-Z. (2007). A study of how market knowledge management capability affects operating performance: from a dynamiccapability point of view, MA, Graduate Institute of Management, National Chiayi University.

29) Jöreskog, K. G., \& Sörbom, D. (2006). LISREL (Version 8.80) [Computer software]. Lincolnwood, IL: Scientific Software International.

30) Kamasak, R. and Bulutlar, F. (2010), “The influence of knowledge sharing on innovation", European Business Review, Vol. 22 No. 3, pp. 306-317.

31) Koch, A. (2011). Firm-internal knowledge integration and the effects on innovation. Journal of Knowledge Management, 15(6), 984-996.

32) Lee, H., \& Choi, B. (2003). Knowledge management enablers, processes, and organizational performance: an integrative view and empirical examination. Journal of 
Management Information Systems, 20(1), 179228.

33) Lee, J.N. (2001), “The impact of knowledge sharing, organizational capability and partnership quality on IS outsourcing success", Information \& Management, Vol. 38 No. 5, pp. 323-335.

34) Liao, S.H., Fei, W.C. and Chen, C.C. (2007), "Knowledge sharing, absorptive capacity and innovation capability: an empirical study of Taiwan's knowledge-intensive industries", Journal of Information Science, Vol. 33 No. 3, pp. 340-359.

35) Lin H F (2006), “Impact of Organizational Support on Organizational Intention to Facilitate Knowledge Sharing", Knowledge Management Research and Practice, Vol. 4, No. 1, pp. 26-35.

36) Lin H F (2007), "Knowledge Sharing and Firm Innovation Capability: An Empirical Study", International Journal of Manpower, Vol. 28, Nos. 3 \& 4, pp. 315-332

37) Lin H F and Lee G G (2004), "Perceptions of Senior Managers Toward Knowledge Sharing Behavior", Management Decision, Vol. 42, No. 1, pp. 108-125.

38) Martelo-Landroguez, S. and Cegarra-Navarro, J.G. (2014), "Linking knowledge corridors to customer value through knowledge processes", Journal of Knowledge Management, Vol. 18 No. 2, pp. 342-365.

39) Martín-De Castro, G., López-Sáez, P. and Delgado-Verde, M. (2011), “Towards a knowledge-based view of firm innovation: theory and empirical research", Journal of Knowledge Management, Vol. 15 No. 6, pp. 871874.

40) Matin H Z, Alvani S M, Jandaghi G R and Pashazadeh Y (2010), “Designing and Clarifying Knowledge Sharing Model in Administrative Agencies to Improve the Performance", European Journal of Economics, Finance and Administrative Sciences, No. 22, pp. 101-111.

41) Matzler, K., Renzl, B., Renzl, B., Mooradia, T., Krogh, G., \& Mueller, J. (2011). Personality traits, affective commitment, documentation of knowledge, and Knowledge Sharing. The International Journal of Human Resource Management, 22(2), 296-310.

42) Ngah R and Ibrahim A R (2010), "The Effect of Knowledge Sharing on Organizational Performance in Small and Medium Enterprises", available at http:// www.kmice.cms.net.my/ProcKMICe/KMICe20 10/Paper/PG467_472.pdf.

43) Nonaka I (1994), “A Dynamic Theory of Organizational Knowledge Creation", Organization Science, Vol. 5, No. 1, pp. 14-37.

44) Nonaka I and Takeuchi H (1995), The Knowledge-Creating Company: How Japanese Companies Create the Dynamics of Innovation, Oxford University Press, New York, USA.

45) Nonaka, I., Toyama, R. and Nagata, A. (2000), “A firm as a knowledge-creating: a new perspective on the theory of the firm", Industry and Corporate Change, Vol. 9 No. 1, pp. 1-20.

46) Ogunseye, O. S., Adetiloye, P. K., Idowu, S. O., Folorunso, O. \& Akinwale, A. T. (2011). Harvesting knowledge from computer mediated social networks. The Journal of Information and Knowledge Management Systems, 41(3), 252-264.

47) Peng, G. C., and Nunes, M. B. 2010. “Why ERP Post-Implementation Fails? Lessons Learned from A Failure Case in China," in Proceedings of the 14th Pacific Asia Conference on Information Systems, Taipei, Taiwan, July 9-12.

48) Podsakoff, P. M., MacKenzie, S. B., Lee, J.-Y., and Podsakoff, N. P. 2003. "Common Method Biases in Behavioral Research: A Critical Review of the Literature and Recommended Remedies," Journal of Applied Psychology (88), pp. 879-903.

49) Polanyi M (1966), The Tacit Dimension, Anchor Day Books, New York.

50) Roberts J (2000), “From Know-How to ShowHow? Questioning the Role of Information and Communication Technologies in Knowledge Transfer", Technology Analysis and Strategic Management, Vol. 12, No. 4, pp. 429-443

51) Sanchez, R., \& Mahoney, J. T. (1996). Modularity, flexibility, and knowledge management in product and organization design. Strategic 
Management Journal, 17(S2), 63-76.

52) Spender J C (1996), “Organizational Knowledge, Learning and Memory: Three Concepts in Search of a Theory", Journal of Organizational Change Management, Vol. 9, No. 1, pp. 63-78.

53) Sugarman, B. (2010). Magic from social networks that talk to management: four cases. The Learning Organisation, 17(4), 288-302.

54) Todorova, G. and Durisin, B. (2007), “Absorptive capacity: valuing a reconceptualization", Academy of Management Review, Vol. 32 No. 3, pp. 774-786.

55) Tsoukas H (1996), “The Firm as a Distributed Knowledge System: A Constructionist Approach", Strategic Management Journal, Vol. 17, Winter, pp. 11-25

56) Tuntrabundit, K. and Tuntrabundit, V. (2011), "Antecedents and consequence of alliance formation", European Journal of Management, Vol. 11 No. 3, pp. 1-7.

57) Turel, O., \& Bart, C. (2014). Board-level IT governance and organizational performance. European Journal of Information Systems, 23(2), 223-239.

58) Uncles M D (2011), "Researching Market Orientation and Business Performance", Australasian Marketing Journal, Vol. 19, No. 3, pp. 161-164; as Quoted in Farooq R (2014), “A Clever Approach to Measure Organizational Performance: An Overview", Prabandhan: Indian Journal of Management, Vol. 7, No. 5, pp. 34-46.

59) Vij S and Bedi H S (2012), “Relationship Between Entrepreneurial Orientation and Business Performance: A Review of Literature", The IUP Journal of Business Strategy, Vol. 9, No. 3, pp. 17-31.

60) Wang $Z$ and Wang N (2012), "Knowledge Sharing, Innovation and Firm Performance", Expert Systems with Applications, Vol. 39, No. 10, pp. 8899-8908.

61) Wang, C. -C. (2004). The influence of ethical and self-interest concerns on knowledge sharing intentions among managers: An empirical study. International Journal of Management, 21(3),
370-381.

62) Wasko M M and Faraj S (2000), "It Is What One Does: Why People Participate and Help Others in Electronic Communities of Practice", Journal of Strategic Information Systems, Vol. 9, No. 2, pp. 155-173.

63) Wasko M M and Faraj S (2005), “Why Should I Share? Examining Social Capital and Knowledge Contribution in Electronic Networks of Practice", MIS Quarterly, Vol. 29, No. 1, pp. 35-57.

64) Wu W Li, Yeh R S and Hung H K (2012), "Knowledge Sharing and Work Performance: A Network Perspective", Social Behavior and Personality, Vol. 40, No. 7, pp. 1113-1120.

65) Yoo Y, Lyytinen K and Heo D (2007), “Closing the Gap: Towards a Process Model of PostMerger Knowledge Sharing", Information Systems Journal, Vol. 17, No. 4, pp. 321-347. 